\title{
Controlling Ambient Information Flow Between Smart Objects with a Mobile Mixed-Reality Interface
}

\author{
Bastian Kriesten \\ Christian Mertes \\ René Tünnermann \\ Thomas Hermann \\ Cognitive Interaction Techology - Center of Excellence (CITEC) \\ Bielefeld University, Germany \\ \{bkrieste,rtuenner,cmertes,thermann\}@techfak.uni-bielefefeld.de
}

\begin{abstract}
In this work we present a method to intuitively issue control over devices in smart environments, to display data that smart objects and sensors provide, and to create and manipulate flows of information in smart environments. This makes it easy to customize smart environments by linking arbitrary data sources to various display modalities on the fly. Touchscreen smartphones - as readily available multipurpose devices - are used to overlay real objects with virtual controls. We evaluated this system with a first qualitative user study.
\end{abstract}

\section{Categories and Subject Descriptors}

H.5.1 [Information Interfaces and Presentation]: Multimedia Information Systems-Artificial, augmented, and virtual realities; J.9.a [Mobile Applications]: Location-dependent and sensitive

\section{General Terms}

Human Factors, Design

\section{Keywords}

Mixed reality, augmented reality, mobile interaction, mobile devices, home automation, ambient data streams

\section{INTRODUCTION}

We are becoming ever more overwhelmed by information from sources such as mail, instant messaging, RSS, and Twitter as well as from the growing number of sensors in our environment, be they in mobile devices or embedded in our living spaces. Along with the emission of data from such sources come an increasing number of output devices and potential data sinks. We propose a system to dynamically connect these data sources and sinks using a mixed-reality view on a touchscreen smartphone, supporting the flexibility in system behaviour deemed crucial by Davidoff et al. [2]. We implemented the possibility to grab any shown data or control element in the mixed reality view and drop it on any other smart object. This is used to establish persistent channel connections as well as for one-off data transfers. Information readout and device control can therefore be done either by

Copyright is held by the author/owner(s).

MobileHCI 2010 September 7-10, 2010, Lisboa, Portugal.

ACM 978-1-60558-835-3.
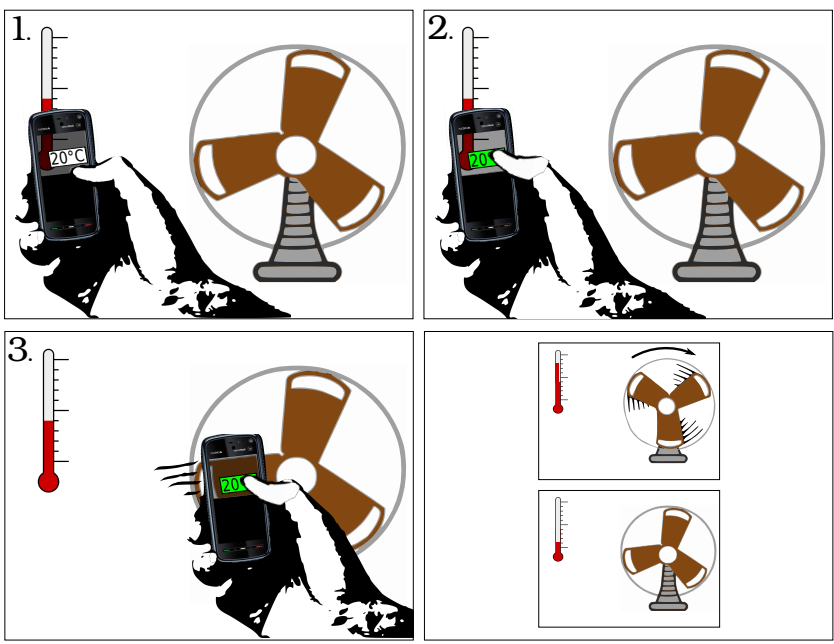

Figure 1: Illustration of the ad-hoc creation of a temperaturecontrolled fan.

using the mobile phone itself or via objects in the smart environment, previously establishing connections with the physical drag and drop gesture. So when a user wants to create a temperature-controlled fan, they can just grab the temperature readings from a thermometer and drop them onto the fan (Figure 1). ${ }^{1}$

\section{RELATED WORK}

Parts of our work were inspired by Rukzios [4] and Rohs [3], the latter of which also wrote the visual marker recognition we used. Using an augmented video stream to observe and control objects was introduced by Tani et al. [6]. This also inspired a very recent system called Touch Projector by Boring et al. [1]. Seifried et al. [5] proposed CRISTAL in which an interactive surface showing a live video view from above the smart space is used to control a room.

Touch Projector enables remote screen interaction including file dragging and dropping between different screens using touch phones. Boring et al. describe a couple of improvements they did to the original system by Tani et al. that our application could probably profit from as well.

CRISTAL offers a central, large and convenient interface for

\footnotetext{
${ }^{1}$ For demonstration videos of the system see http://www.techfak.uni-bielefeld.de/ags/ami/ publications/KTMH2010-CAI/.
} 
room control, whereas our system runs on mobile devices that people have handy at most times and that can be used to interact with smart objects in the immediate vicinity. Each interaction style might be more useful in different situations.

\section{SYSTEM OVERVIEW}

We designed configurable channel interactions for (1) text and number data labels or input forms, (2) numbers in an interval that are shown as a progress bar or - in case of input modalities - as a slider widget (an alternative implemented input method for this is using physical rotation of the mobile phone similar to a tap), (3) signals and on/off boolean values that are shown as either stateless or toggle buttons, (4) vector graphics that are drawn onto the physical object, (5) linked image files that are displayed on the physical object, (6) linked sound data, (7) linked files and websites whose file name or URL is shown. Each physical object is identified by its visual marker ID and represented by a software module in a decentralized network. Modules communicate using the publish/subscribe paradigm on $\mathrm{XMPP}^{2}$. The marker recognition is done on the mobile device itself.

\section{EVALUATION}

We evaluated our prototype system with 9 subjects, 6 male and 3 female, ranging from 23 to 35 years in age with the mean age being 28.8 years.

We chose the following set of smart objects to be made available: a digital picture frame, a thermometer, LED lights, a fan, chains of lights, a loudspeaker, and CD covers. The subjects had to read instructions consisting of five steps: (1) general system familiarization (2) object control (picture frame) (3) data readout (thermometer) (4) file transfer (music to speakers) (5) object connection (temperature to LED light color). Each step encompassed a conceptual explanation and a concrete task which had to be completed before moving on to the next step. The subjects were observed and videotaped during the interaction. After performing the tasks, subjects were allowed to continue exploring the system. They were finally asked to fill in a questionnaire with five multiple choice questions and seven free response questions.

\subsection{Results}

The following table shows the results of the multiple choice questions. Each question had four options, 1 being most positive (options 1 and 4 bore question-specific labels):

How intuitive did you find the system?

How quickly did you get used to the system?

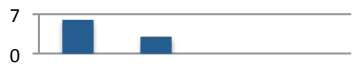

How likely is it for you to actually use such a system?

How efficient to use did you find the system?

How pleasant ("angenehm") to use did you find the system?
For the free responses, the issue raised the most was the jitter in the marker recognition. This point was brought up by 7 subjects. The size of the display or the controls was criticized by 5 subjects and it was mentioned by 4 subjects that they would have liked the system to work at a greater distance to be more like a remote control. Connecting different devices was highlighted as a particularly exciting possibility by 5 of the subjects. Six subjects said that the system was fun to use or showed that by spontaneous smiling or laughing or by exclamations such as "cool" or "awesome".

\subsection{Discussion}

One of our main goals was to allow for intuitive and direct interaction. The described evaluation indicates that this goal was achieved. The flexibility that arises from being able to freely connect various devices and channels also was received well by most of our test subjects. On the other hand, the study pointed out the direction in which further development is most promising. This is mainly tackling the difficult handling of the mobile phone, namely the small, moving and sometimes flickering controls, because the users had difficulties in focusing the objects and keeping the smartphone still while touching the mixed reality elements on the screen. We think this can also explain the discrepancy between the relatively low effectiveness rating compared to the good intuitiveness rating.

\section{CONCLUSION}

We proposed a way to control, query and especially interconnect smart objects with a mixed reality smartphone application that uses visual markers to identify objects. With our system based on a mobile mixed reality interface we introduced a method to make digital and virtual data and data streams graspable and manageable.

We showed the system to be useful and accepted by subjects despite there being a couple of technical difficulties that will need further improvement. We believe the combination of mixed reality, mobile phones and ambient information flow to be a promising approach for home automation and ambient intelligence - in itself as well as in combination with other interfaces.

\section{REFERENCES}

[1] S. Boring, D. Baur, A. Butz, S. Gustafson, and P. Baudisch. Touch projector: Mobile interaction-through-video. In Proc. of the 2010 International Conference on Human Factors in Computing Systems, New York, USA, Apr. 2010. ACM.

[2] S. Davidoff, M. Lee, C. Yiu, J. Zimmerman, and A. Dey. Principles of smart home control. UbiComp 2006: Ubiquitous Computing, pages 19-34, 2006.

[3] M. Rohs. Linking Physical and Virtual Worlds with Visual Markers and Handheld Devices. PhD thesis, Swiss Federal Institute of Technology Zurich, 2005.

[4] E. Rukzio. Physical Mobile Interactions: Mobile Devices as Pervasive Mediators for Interactions with the Real World. $\mathrm{PhD}$ thesis, Ludwig-Maximilians-Universität München, Dec. 2006.

[5] T. Seifried, M. Haller, S. D. Scott, F. Perteneder, C. Rendl, D. Sakamoto, and M. Inami. Cristal: A collaborative home media and device controller based on a multi-touch display. In Proc. of the International Conference on Interactive Tabletops and Surfaces 2009, pages 37-44, New York, USA, 2009. ACM.

[6] M. Tani, K. Yamaashi, K. Tanikoshi, M. Futakawa, and S. Tanifuji. Object-oriented video: interaction with real-world objects through live video. In Proc. of the 1992 Conference on Human Factors in Computing Systems, pages 593-598, New York, USA, 1992. ACM.

\footnotetext{
${ }^{2}$ http://xmpp.org/
} 